Perceived vaccine safety over time in a vaccine hesitant sample: Impact of pausing due to safety concerns

Thomas L. Rodebaugh

Madelyn R. Frumkin

Rachel Garg

Laura LaGesse

Amy McQueen

Matthew W. Kreuter

Washington University in St Louis

Author Note

Thomas L. Rodebaugh and Madelyn R. Frumkin, Department of Psychological and Brain

Sciences, Washington University in St Louis. Rachel Garg, Laura LaGesse, and Matthew W.

Kreuter, Health Communication Research Laboratory (HCRL), Brown School, Washington

University in St Louis. Amy McQueen,HCRL and School of Medicine, Washington University

in St. Louis. The study was supported by a competitive supplement to a research grant from the

National Cancer Institute (R01CA201429). Correspondence: Thomas L. Rodebaugh, 1

Brookings Drive, Campus Box 1125, Somers Family Hall, Department of Psychological and

Brain Sciences, Washington University in St Louis, St. Louis, MO 63130; email:

rodebaugh@wustl.edu

THIS MANUSCRIPT HAS BEEN SUBMITTED FOR PUBLICATION. THIS FILE

CONTAINS THE MANUSCRIPT AND ITS SUPPLEMENT. IT IS NOT A FINAL PEER-

REVIEWED MANUSCRIPT.

Supplemental materials: https://osf.io/7ti5c/ 


\begin{abstract}
In the current effort to vaccinate as many people as possible against COVID-19, it has been suggested that events such as the pause in the use of the Janssen vaccine would have a large effect on perceptions of vaccine safety. Further, as vaccination rates slow, there is concern that hesitancy may be stable and difficult to change among those still unvaccinated. We examined both of these issues in our ongoing study of low-income participants. We modeled the intensive longitudinal data provided by 53 individuals. We found the negative, not statistically significant effect of the Janssen pause would be overwhelmed within weeks by the statistically significant increasing perceptions of safety across time. We also observed strong variability in vaccine hesitancy in many participants. Frequent reminders about vaccine availability might catch more people when they are less hesitant, helping increase vaccination rates.
\end{abstract}

Keywords: COVID-19; vaccination; vaccine hesitancy; Janssen vaccine 


\section{Perceived vaccine safety over time in a vaccine hesitant sample: Impact of pausing due to safety concerns}

National polling shows that public confidence in COVID-19 vaccines is rising in the U.S., (AP-NORC Research, 2021), and the impact of the Janssen (i.e., Johnson \& Johnson) vaccine pause on vaccine confidence and uptake was minimal (de Beaumont Foundation, 2021), 2021; Axios-Ipsos, 2021). It is unclear whether these general population polling resalts hold true for underserved Americans. News reports indicated the Janssen pause could deepen skepticism among low-SES Americans already hesitant to get vaccinated (Glenza, 2021), whereas past research suggests that many in these groups may not even be aware of the pause.

Studies show that even during long periods of intense media attention and scrutiny, the reach and impact of news about major changes to health and medical guidelines varies by SES. Surveys administered in the midst of highly publicized controversies about breast cancer screening guidelines find that awareness of the controversy is consistently lower among women with fewer years of education and lower income (Kiviniemi \& Hay, 2012; Kreuter et al., 2004; Meissner et al., 2003). Public awareness of past changes to vaccine recommendations also appears to be greater in some groups than others. For example, parents of boys seen at private pediatrics offices were more likely than parents of boys seen at public health clinics to know that the HPV vaccine had been approved for boys (Tan \& Gerbie, 2017).

We performed a preliminary analysis of data from a unique ongoing study among lowincome women, Life During COVID. In the study, we collect intensive longitudinal data administering surveys by mobile phone every three days - assessing perceptions of vaccine safety, among other life experiences. Data were collected before and after the Janssen vaccine pause, allowing us to test: (1) whether the pause affected perceived vaccine safety beliefs above 
and beyond the effects of time; (2) the stability of vaccine safety beliefs; and, in an exploratory analysis, (3) whether and how self-reported likelihood of being vaccinated shifted over time.

\section{Method}

Additional details of methodology are available in the supplemental material.

\section{Participants}

Our 53 participants were adult women with at least one school-age child and were either employed or looking for work. They were originally identified because they called a community helpline seeking assistance with basic needs and had later completed participation in a smoking cessation trial (McQueen et al., 2019). Those who met initial eligibility criteria were contacted by telephone by a study team member and screened for eligibility. Participants provided verbal consent to participate in the study.

\section{Procedure}

All study materials and procedures were approved by the Institutional Review Board at Washington University in St. Louis. After participants completed an initial telephone interview, a survey link was texted to their mobile phone every three days. The survey included all items analyzed here, among others.

\section{Measures}

Participants rated COVID-19 vaccine safety ("From what you've heard, how safe do you think the vaccine is?"; from 0, Not at all safe, to 100, Extremely safe), with no distinction made between different vaccines; whether they had been vaccinated (yes or no), and, if they had not, their likelihood of taking the vaccination ("If the vaccine was available to you today, how likely would you be to take it?"; from 0, Not at all likely to 100, Extremely likely).

\section{Quantitative Procedure}


Dynamic structural equation models (DSEM) were conducted in Mplus. We used the multilevel model for safety concerns, and $N=1$ models for vaccine likelihood. These Bayesian models for intensive longitudinal data are similar to more familiar multilevel models, but have greater flexibility (Asparouhov et al., 2018; Asparouhov \& Muthén, 2020; McNeish \& Hamaker, 2020). All multilevel models included random slopes, intercepts, and residuals.

Vaccine safety perceptions were predicted by (a) whether the Janssen vaccine had been paused $(0=$ survey completed prior to April 13th; $1=$ survey completed later $)$ and (b) the previous survey's value for vaccine safety. In addition, survey number was included as a predictor. Including this timing variable allowed us to partial out any linear effects related to time. Whether perceptions of vaccine safety were increasing or decreasing overall was also of interest. For $N=1$ models of vaccination likelihood, the outcome variable was predicted by itself and time.

\section{Results}

Fifty-three participants completed at least 1 survey between February $20^{\text {th }}, 2020$ and May $25^{\text {th }}$, 2021. Participants' mean age was $36.6(\mathrm{SD}=8.3)$ years. Most were African American $(81 \%)$ or White $(19 \%), 23 \%$ had completed $\leq 12$ years of schooling, $57 \%$ reported annual pre-tax household income of $\varangle \$ 20,000$, and $66 \%$ had multiple children at home. Three in five (60\%) were essential-workers and 23\% were looking for work. At the time of study enrollment, $46 \%$ reported that they were either unlikely $(42 \%)$ or unsure (4\%) about getting vaccinated against CONID-19.

\section{Modeling Effects of Vaccine Pause}

Group effects. Full model syntax and detailed results are available in the supplemental material. The model selection process led us to concentrate on the ML-DSEM model. In short, 
the confidence interval for the effect of the Janssen pause was largely negative but not statistically significant, which suggests that although it is very unlikely the pause increased perceived vaccine safety, any negative impact was not reliably detected. The only statistically significant $(p<.05)$ finding was an effect for perceived safety increasing over time. Comparing the size of these two effects revealed that even if the effect of the pause were meaningful, it would be overcome by the time effect before 9 days had passed. In a post-hoc test reported in the supplemental material, we tested whether participants' own vaccine status might account for any of these results; they did not.

A general picture of perceptions about vaccine safety across time can be seen in Figure 1, which depicts average vaccine safety ratings both relative to the vaccine pause announcement bars and, for those participants providing more than ten surveys, across time trend lines. The figure thus clearly depicts an at-best negligible effect of the Janssen pause, but a clear effect of time on increased perceptions of safety.

Individual effects. Vaccine pause did not demonstrate a significant result for any individual. In contrast, the time variable was significant and positive for 10 participants, significant and negative for one, and not significant for the remaining 42 .

\section{Vaccine Hesitancy}

In Figure 2, we depict the time course of ratings of the likelihood of getting a COVID vaccine among all individuals who had not yet been vaccinated and provided at least ten data points. The first four participants showed no variation in their ratings. The rest all show significant variation across time. Two showed significant increases in likelihood over time, whereas one showed a significant decrease.

\section{Discussion}


We tested whether the Janssen vaccine pause had any strong effect on perceptions of vaccine safety in our ongoing study of low-income women. Instead of finding such an effect, we found a significant tendency for beliefs about vaccine safety to rise over time. Among participants who had not been vaccinated for COVID-19, we also examined over time their selfrated likelihood of getting vaccinated. These ratings displayed clear variability for many participants.

Our results must be considered in the context of several important limitations of this ongoing study. Our sample is relatively small at this point, both in terms of participants and time points. It is possible that there was an effect of the Janssen pause, but it was too small for us to detect reliably; however, this possibility still leaves the significant time effect we observed as a more important effect. It is also possible that our results would not generalize to other lowincome populations of interest to public health researchers, for example, men, older adults, and those without school-age children. However, if we assume that our results would generalize, but that the effect of the Janssen pause was small, many commonly used study methodologies - such as asking participants before and after an event - would also fail to find the effect. In fact, our results indicate that conventional methods might lead to the erroneous conclusion that the vaccine pause increased perceptions of vaccine safety, whereas our results indicate that such a finding is due to gradual increases in perceptions of safety as a function of time.

Our results have several implications for health education and public health efforts to reduce vaccine hesitancy. First, the findings suggest that individual news stories or events, although felt acutely by experts, may not have much or lasting impact on the impressions of individuals who are not highly focused on these questions. Instead, gradual changes over time may be driven by secular trends and accumulated evidence that individuals gain from a variety of 
sources. Second, our results challenge the idea that vaccine hesitancy is a relatively stable and unchangeable construct. This appeared to be true for some, but not all of our participants. In fact, for some participants, changing vaccine hesitancy may not even be necessary to change their willingness to be vaccinated. Frequent reminders about vaccine availability might catch such people when they are less hesitant, helping increase vaccination rates. 


\section{References}

AP-NORC Research (2021). Confidence in COVID-19 Vaccines Rises. Retrieved June 15 from apnorc.org/projects/confidence-in-covid-19-vaccines-rises/

Asparouhov, T., Hamaker, E. L., \& Muthen, B. (2018). Dynamic structural equation models. Structural Equation Modeling, 25(3), 359-388. https://doi.org/10.1080/10705511.2017.1406803

Asparouhov, T., \& Muthén, B. (2020). Comparison of models for the analysis of intensive longitudinal data. Structural Equation Modeling: A Multidisciplinary Journal, 27(2), 275-297.

Axios-IPSOS. (2021). America's reopening shows little signs of slowing down. Retrieved June 15, 2021 from https://www.ipsos.com/en-us/news-polls/axios-ipsos-coronavirus-index de Beaumont Foundation (2021). Poll: Despite Johnson \& Johnson pause, vaccine confidence is rising, while partisan divide on vaccination is narrowing. Retrieved June 15, 2021 from https://debeaumont.org/changing-the-covid-conversation/jj/

Glenza, J. (2021, 19 April 2021). Fear that Johnson \& Johnson pause could heighten vaccine hesitancy in US. The Guardian.

https://www.theguardian.com/society/2021/apr/19/johnson-johnson-pause-vaccinehesitancy-us

Kiviniemi, M. T., \& Hay, J. L. (2012). Awareness of the 2009 US Preventive Services Task Force recommended changes in mammography screening guidelines, accuracy of awareness, sources of knowledge about recommendations, and attitudes about updated screening 
guidelines in women ages 40-49 and 50+. BMC Public Health, 12, 899.

https://doi.org/10.1186/1471-2458-12-899

Kreuter, M. W., Holt, C. L., \& Skinner, C. S. (2004). Awareness of mammography controversy among lower-income African American women in urban public health centers. Journal of Womens Health (Larchmt), 13(1), 121-122. https://doi.org/10.1089/154099904322836546

McNeish, D., \& Hamaker, E. L. (2020). A primer on two-level dynamic structural equation models for intensive longitudinal data in Mplus. Psychological Methods, 25(5), 610-635. https://doi.org/10.1037/met0000250

McQueen, A., Roberts, C., Garg, R., Caburnay, C., Fu, Q., Gordon, Y., Bush, T., Pokojski, R., Thompson, T., \& Kreuter, M. (2019). Specialized tobacco quitline and basic needs navigation interventions to increase cessation among low income smokers: Study protocol for a randomized controlled trial. Contemporary Clinical Trials, 80, 40-47. https://doi.org/10.1016/j.cct.2019.03.009

Meissner, H. I., Rimer, B. K., Davis, W.W., Eisner, E. J., \& Siegler, I. C. (2003). Another round in the mammography controversy. Journal of Womens Health (Larchmt), 12(3), 261-276. https://doi.org/10,1089/154099903321667609

Tan, T. Q., \& Gerbie, M. V. (2017). Perception, awareness, and acceptance of human papillomavirus disease and vaccine among parents of boys aged 9 to 18 years. Clinical Rédiatrics (Phila), 56(8), 737-743. https://doi.org/10.1177/0009922816682788 
Figure 1. Depiction of Pause Effect Relative to Time Effect

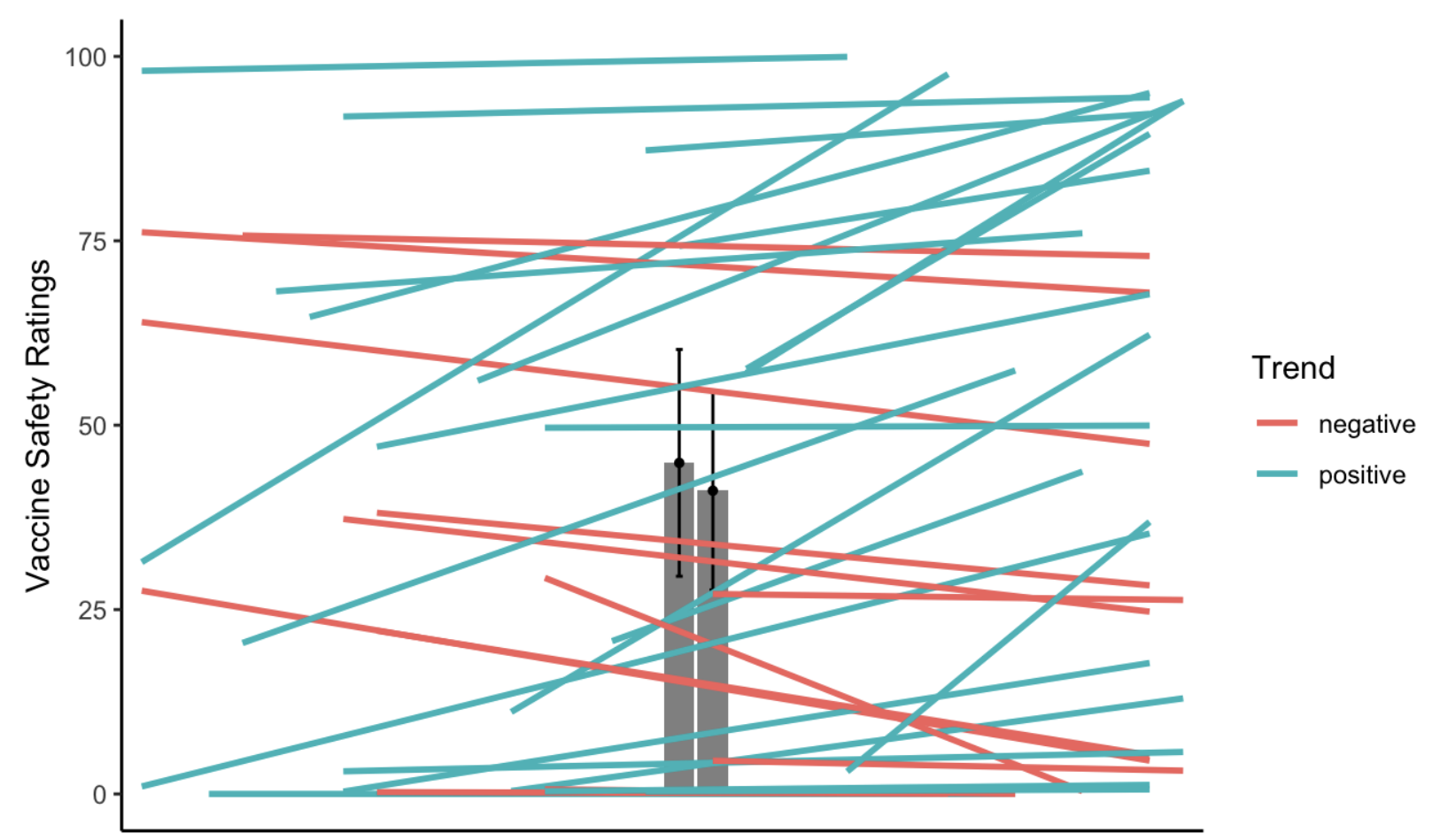

February 20, 2021 to May 25, 2021

Note. The figure depicts both the potential Janssen pause effect (bars) and the time effect (trend lines). The bars show the average of the ratings the three days before the pause (left) and after the Janssen pause (starting the day the pause happened, since the pause occurred in the early morning hours). The error bars show $95 \%$ confidence intervals for the mean. Trend lines are for those participants who had completed 10 or more surveys, with green slopes indicating positive values and red slopes indicating negative values. 
Figure 2. Trace Lines of Reports of Vaccine Likelihood over Time Arranged by Variance

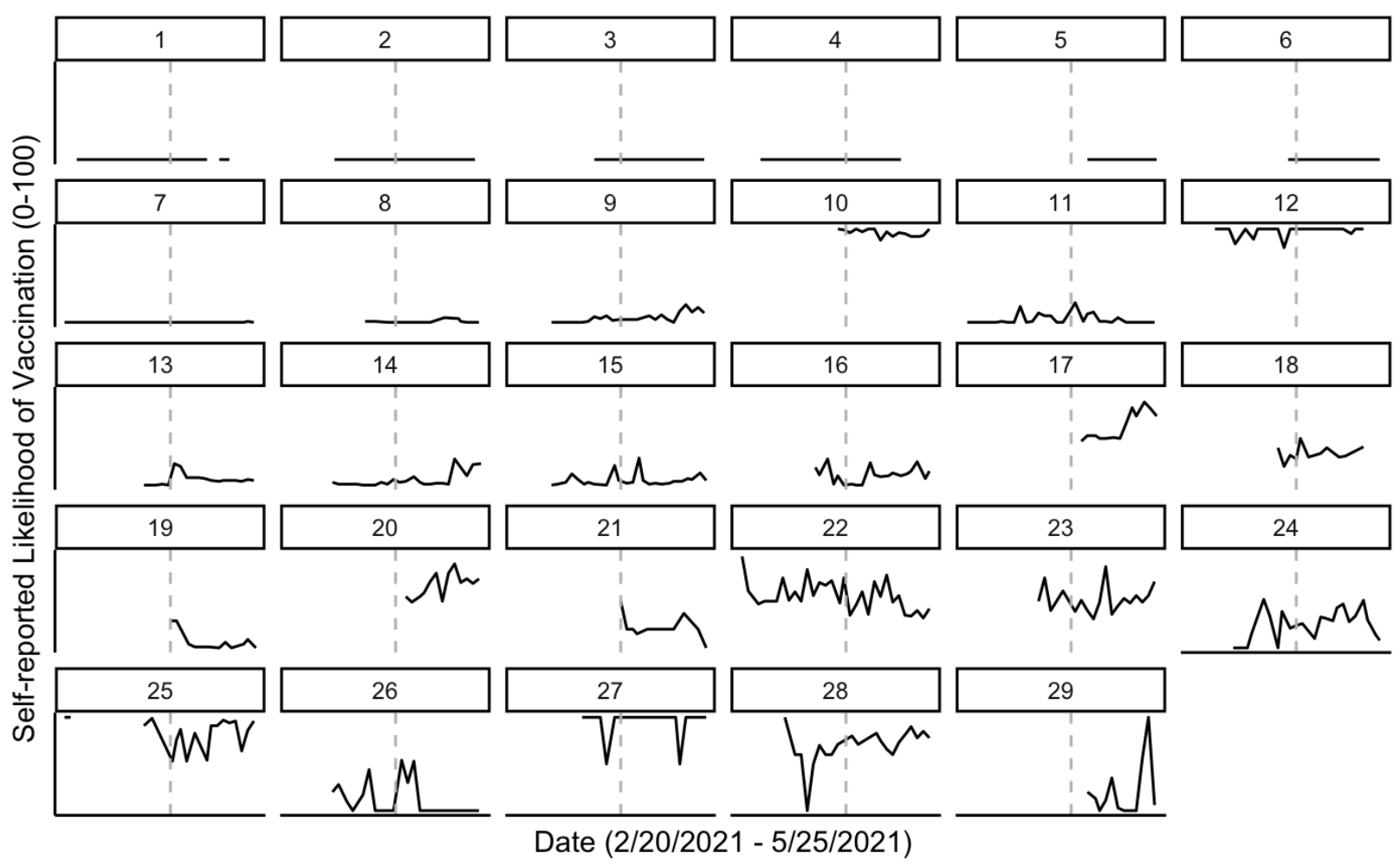

Note. Data from 29 participants who provided 10 or more surveys and had not yet been

vaccinated. Panels are ordered by amount of variance after any trends and autoregressive tendencies are partialled out. The first six participants showed no variation at all in responses. Participant 22 showed a significant decrease over time, whereas Participants 9 and 14 showed significant upward slopes.

Supplemental materials at: https://osf.io/7tj5c/ 